\title{
UCRL-BOOK-207340
}

LAWRENCE LIVERMORE N A T IO N A L

\section{Spectroscopy of ions using fast beams and ion traps}

E.H. Pinnington, E. Träbert

October 19, 2004

Handbook of Atomic, Molecular and Optical Physics 2nd edition 
This document was prepared as an account of work sponsored by an agency of the United States Government. Neither the United States Government nor the University of California nor any of their employees, makes any warranty, express or implied, or assumes any legal liability or responsibility for the accuracy, completeness, or usefulness of any information, apparatus, product, or process disclosed, or represents that its use would not infringe privately owned rights. Reference herein to any specific commercial product, process, or service by trade name, trademark, manufacturer, or otherwise, does not necessarily constitute or imply its endorsement, recommendation, or favoring by the United States Government or the University of California. The views and opinions of authors expressed herein do not necessarily state or reflect those of the United States Government or the University of California, and shall not be used for advertising or product endorsement purposes. 


\title{
Spectroscopy of Ions using Fast Beams and Ion Traps
}

\author{
Eric H. Pinnington ${ }^{\dagger}$ and Elmar Träbert\# \\ $\dagger$ Department of Physics, University of Alberta, \\ Edmonton, Alberta, T6G 2J1 Canada \\ \# Fakultät für Physik und Astronomie, \\ Ruhr-Universität Bochum, D-44780 Bochum, Germany, and \\ High Temperature and Astrophysics Division, \\ Lawrence Livermore National Laboratory, Livermore, CA 94550, U.S.A.
}

PACS numbers:

This work was performed under the auspices of the U. S. Department of Energy by University of California, Lawrence Livermore National Laboratory under contract W-7405-Eng-48. 


\section{INTRODUCTION}

A knowledge of the spectra of ionized atoms is of importance in many fields (see Sect. 17.1). They can be studied in a wide variety of light sources. In recent years techniques coming under the broad headings of fast beams and ion traps have been used extensively for such investigations. This article will consider the advantages that various techniques have for particular applications.

\section{SPECTROSCOPY USING FAST ION BEAMS}

A beam of ionized atoms has several advantages as a spectroscopic source. Unlike arcs, sparks and high temperature plasmas, the ions can be studied in an environment that is free of electric and magnetic fields and relatively free of interparticle collisions [1]. Standard accelerator techniques can be used to produce a well-collimated, mass-analyzed beam of ions having a low velocity spread. In principle, virtually any charge state of any element, isotopically pure if required, can be obtained. Finally, the well-defined velocity of the ions permits the study of processes evolving in time in terms of their spatial evolution along the beam. This is particularly important in the case of lifetime measurements.

\section{A. Beam-Foil Spectroscopy}

A beam of ions passing through a thin $(50-200 \mathrm{~nm})$ foil emerges in a range of ionization states, with the mean charge state increasing with the incident energy [2]. Thin foils made from a light element, usually carbon, are used to minimize particle scattering and energy straggling. Thus, a $\mathrm{F}^{+}$beam of $0.5 \mathrm{MeV}$ that enters a carbon foil emerges from it

with a mean charge of about $+2 \mathrm{e}$, while a Xe ion beam at $180 \mathrm{MeV}$ emerges with a mean charge of about $+29 \mathrm{e}$. The outer electrons are distributed over many different states, most of which then decay to lower states by photon emission as the ions move away from the foil. The beam-foil interaction is highly non-selective, which is an advantage for spectroscopic studies but causes a problem for lifetime measurements. (Methods for tackling this problem are discussed in Sect. 17.3.2.) A major disadvantage of the beam-foil light source is its low intensity; consequently scanning spectrometers have usually been equipped with photon-counting detectors. However, in recent years position sensitive detectors have be- 
come available, which permit the simultaneous recording of information over a wide spectral range, resulting in a greatly improved detection efficiency (see Sect. 17.3.4).

The intrinsic properties of the beam-foil interaction are important in understanding its usefulness for spectroscopic studies. The electrons of the moving ion are shielded from the travelling ion core as the ion passes through the foil and are then recaptured into a statistical distribution of outer states. The probability that more than one electron in a given ion will be captured in an excited state is high relative to other light sources, and hence the technique has been used extensively to study doubly and multiply excited states [3] (see Sect. 62.1.1?). The interaction also favours the production of high- $L$ Rydberg states [4] (see Sect. 14.1?).

At low incident ion energies, electron capture can give a downstream beam containing neutral and even negative ions. The first observation of photon emission between bound states in a negative ion was achieved using beam-foil excitation [5]. A more recent example of multiple excitation in a negative ion is the identification in lithium-like $\mathrm{He}^{-}$of the $2 \mathrm{p}^{3}$ ${ }^{4} \mathrm{~S}^{\circ}$ state in which all three electrons are excited $[6,7]$. The observation of the same triply excited level in neutral Li has resulted in one of the most precise $(40 \mathrm{ppm})$ wavelength measurements in beam-foil spectroscopy [8]. The time-resolution inherent in the beam-foil source can also be used to aid in the identification of transitions from long-lived states, such as intercombination transitions [9] (see Sect. 10.17?). Here the beam foil spectrum is recorded first close to the foil and then far downstream (a few $\mathrm{mm}$ to a few $\mathrm{cm}$ ), "far" in this context meaning that the short-lived states have had time to decay. The intercombination (or other long-lived) transitions are then easily identified by their relatively strong intensity in the (overall much weaker) downstream spectrum. This has been a decisive step in identifying laboratory lines with lines in the solar corona $[10,11]$. A recent example of beam-foil spectroscopic analysis is given in Ref. [12].

One problem with using fast ions as a light source for precision wavelength measurements is the inevitable Doppler effect broadening of the spectral lines. However, this can be removed by appropriately refocusing the spectrometer [13, 14]. Furthermore, since the Doppler width varies with the wavelength, and the line width of a given grating spectrometer used on a fast ion beam usually is dominated by spectrometer geometry, not by diffraction, Doppler broadening often becomes less important in measurements at shorter wavelengths, as in the UV or EUV. This is the primary range of emission from the more highly-ionized atoms, and that is where beam-foil spectroscopy really comes into its own. For example, the 
leading terms omitted in calculations of the wavelength of the $1 \mathrm{~s} 2 \mathrm{~s}{ }^{3} \mathrm{~S}-1 \mathrm{~s} 2 \mathrm{p}{ }^{3} \mathrm{P}^{\circ}$ transition in He-like ions [15] scale as $\mathrm{Z}^{4}$, with the result that measurements made with higher-Z ions need not have as high a precision for a meaningful test of the calculation as would be required for a low-Z ion. The beam-foil measurement for the leading ( $\mathrm{J}=1$ to $\mathrm{J}=2$ ) component in $\mathrm{Ni}^{26+}[16]$ has an uncertainty of $0.02 \%$, which is about equal to that in the calculation. The two values agree well within this limit. (Naturally, measurements made using the beam-laser techniques discussed in Sect. 18.2.3 yield results with a much higher precision, but such measurements are restricted to low-Z ions because of the excitation energy steps and transition wavelengths involved, and here the theoretical uncertainties are also much smaller.)

Turning now to beam-foil lifetime measurements, here the intensity of a given transition is studied as a function of the time that has elapsed since excitation, usually by stepping the foil upstream. Because of the non-selective nature of the excitation, the possibility exists that the state being studied is itself being repopulated by higher-lying states, resulting in a decay curve that consists of the sum of exponential terms, one term corresponding to the primary level being studied and the other terms to higher-lying levels involved in repopulating that level. The analysis of such decay curves can be problematic. Several computer routines have been developed to tackle this problem, such as DISCRETE [17] and HOMER [18]. One useful trick here is to record the decay curve for each of the major transitions repopulating a given primary level and then include the lifetimes obtained for those transitions as fixed parameters in fitting the decay curves for that primary level. A more rigorous method to include the decay data from the repopulating transitions in the analysis of the primary lifetime is the ANDC technique described in detail in Section 17.3.2. An additional problem may arise in the measurement of very short lifetimes, which tend to be associated with short-wavelength transitions for which no lenses are available to focus the beam at the spectrometer entrance slit. The observation region is then defined by the spectrometer aperture and extends for a finite length along the beam that can be comparable to, or even greater than, the decay length for that transition. Here it is necessary to fit the decay curve including the vignetted region around the foil $[19,20]$. Lifetimes in the picosecond regime have been successfully measured in this way. 


\section{B. Beam-Gas Spectroscopy}

While the use of a gas target in place of a foil has the obvious advantage that it cannot break, the loss of a tightly-localized excitation region means that the fine time resolution of the beam-foil light source is largely lost. The beam-gas source has two main advantages, however. First, the passage of a beam of fast, highly-stripped ions through a neutral gas results in the production of recoil ions (see Sect. 63.3?), which are moving very slowly relative to the beam ions, thus reducing the Doppler broadening problem mentioned earlier. Secondly, it is possible to study details of the interaction between the gas and beam ions, such as charge-exchange reactions, as they occur, and not merely observing their consequences. Such experiments have experienced a resurgence with the advent of the ECR ion source [21]. A recent example of such work is given in Ref. [22].

When a fast beam of highly charged ions passes through a neutral gas, it leaves a trail of ionized gas atoms in its wake. These ions recoil from the interaction region with relatively low velocities $\left(\mathrm{v} / \mathrm{c}=10^{-4}-10^{-5}\right)$. Furthermore, the recoil velocities tend to be restricted to a narrow range of angles approximately perpendicular to the beam direction. Hence, observation of the radiation emitted by the recoil ions transverse to their motion, i.e. along a direction parallel to the beam, gives a very low Doppler width, $\Delta \lambda_{D} / \lambda$ being typically $10^{-6}$, the limit imposed by the thermal motion of the target gas atoms. Recoil ion spectroscopy is therefore a useful procedure for precision wavelength measurements for highly-stripped ions, such as He-like $\mathrm{Ar}^{16+}$ recoil ions produced by a beam of $2 \mathrm{GeV} \mathrm{\textrm {U } ^ { 7 0 + }}$ ions [23]. The energies and charge states of the recoil ions may be determined using standard time-of-flight techniques [24], and detecting the recoil ions in coincidence with their progenitor ions yields information on the dependence of the recoil energy on the details of the ionizing collision [25]. In later developments, the differentially pumped gas target has first been replaced by a supersonic jet target which reduces the thermal motion of the target particles, and then by a cold atom sample in an atom trap; replacing optical detection by position-sensitive fasttiming detectors for all collision products, the technique of COLTRIMS (COLd Target Recoil Ion Momentum Spectroscopy) now can be employed to study the momentum distribution of the collision partners [26].

Measurements made on the projectile ions themselves also yield useful information about electron-capture processes. The strength of such a process is described in terms of its cross 
section (see Sect. 61.2.2?). Recent work has shifted from measurements of the total cross section for electron capture, to more detailed studies of the individual capture channels [27]. Such studies provide much more stringent tests of theoretical models of the ion-atom charge transfer processes. They often involve such techniques as spectroscopy of the optical radiation or of the Auger electrons (see Sect. 25.1.1?) emitted by the ions after electron exchange. A further example is the study by Prior et al [28] of the angular distribution of Auger electrons emitted by doubly-excited states formed in hydrogen-like projectile ions with an energy of $40 \mathrm{keV}$, following double-electron capture from target helium atoms. They found that significant alignment of the magnetic substates of the projectile ions can result from electron capture. Such anisotropies in the Auger electron emission demonstrate the danger of using single-angle measurements to determine cross sections.

\section{Beam-Laser Spectroscopy}

As for the beam-foil source, excitation of a beam of fast ions by a transverse tuneable laser produces the localized excitation required for high temporal resolution. Now, however, the excitation is highly selective, permitting the population of just a single level. The laser-induced-fluorescence (LIF) signal as a function of the distance along the beam from the excitation region is therefore described by a single exponential decay, for which an exact analysis with rigorous error bounds is possible. The restriction to levels that can be accessed by electric dipole (E1) transitions from the ground and metastable levels may be overcome by combining laser excitation with a non-selective mode of excitation, as in beam-gas-laser [29] or beam-foil-laser [30] measurements. A discussion of precision lifetime measurements using laser excitation of a fast beam is given in Sect. 17.3.2. Here the discussion will be limited to precision optical spectroscopy. Examples of precision beam-laser wavelength measurements may be found in Refs. [31] and [32]. A further example is the measurement of the spin-forbidden $1 \mathrm{~s} 2 \mathrm{~s}{ }^{1} \mathrm{~S}_{0}-1 \mathrm{~s} 2 \mathrm{p}^{3} \mathrm{P}_{1}$ interval in $\mathrm{N}^{5+}$ [33], where the experimental value, $986.321(7) \mathrm{cm}^{-1}$ is in agreement with the calculated value, $986.58(30) \mathrm{cm}^{-1}$. An example

of a similar measurement in a molecular ion is given in [34], while a recent study of the hyperfine structure in a rare-earth ion is given in Ref. [35].

A major aim in beam-laser spectroscopy is to minimize the width of the LIF signal. The instrumental linewidth of the laser itself can be reduced to below $1 \mathrm{kHz}$, so that the 
width of the LIF signal is usually dominated by the velocity spread of the ions and by the divergences of the ion and laser beams. The effects of beam divergence can be minimized by using the collinear geometry, in which the ion and laser beams are parallel. If the angle between the ion and laser beams is $\theta$, the Doppler-shifted laser frequency, as measured in the ion's rest frame, is $f_{L}(1-\beta \cos \theta)$, where is $f_{L}$ is the laser frequency and $\beta=\mathrm{v} / \mathrm{c}$ and is much less than unity. Hence the range in frequency resulting from a beam divergence, $\Delta \theta$, is given by $\mathrm{f}_{L} \beta \sin \theta \Delta \theta$, which tends to zero as $\theta$ tends to zero. One disadvantage of the collinear geometry is that, if the laser is brought into resonance with an atomic transition by adjusting the ion velocity and/or the laser frequency, the LIF signal is produced over the entire overlap region between the ion and laser beams. The resonance can be restricted to a desired region by setting the ion velocity to be slightly off-resonance. The velocity can then be adjusted locally for resonance with the laser by passing the ion beam through a Faraday cage electrode to which an adjustable voltage is applied $[36,37]$. The width of the resonance signal here is usually dominated by the spread in the ion velocity, $\mathrm{c} \cdot \Delta \beta$, usually arising in the ion source being used. The width resulting from a given $\Delta \beta$ can therefore be reduced by using a higher ion energy. This is known as kinematic compression. In terms of the ion energy $\mathrm{E}$, the range in the ion energy $\Delta \mathrm{E}$ and the ion mass $\mathrm{M}$, the Doppler width of the LIF signal is given by $\mathrm{f}_{L} \Delta \mathrm{E} /\left(2 \mathrm{Mc}^{2} \mathrm{E}\right)^{1 / 2}$ and thus decreases as $\mathrm{E}$ is increased.

A more significant improvement in frequency resolution is made possible by including $\mathrm{rf}$ resonance in a laser double-resonance experiment. Here the ions are brought into resonance with an off-resonance laser using two separate Faraday cage electrodes. The first resonance depletes the population in the ion state from which excitation occurs, thus weakening the second resonance signal. An rf field is then applied to the ions between the two electrodes. Tuning the frequency of this field over the region that corresponds to fine- or hyperfinestructure intervals in the ion can then repopulate the state from which laser excitation occurs, thus re-establishing the second laser LIF resonance signal. The width of the resonance signal is now determined by the transit-time broadening that results from the finite time spent by the ions in the rf field (see also Sect. 74.6.1?). For example, in the experiments by Sen et al [37] with a beam of ${ }^{131} \mathrm{Eu}^{+}$ions at $1.35 \mathrm{keV}$, the width of the laser-rf double-resonance signal was $59 \mathrm{kHz}$, compared with a width of $45 \mathrm{MHz}$ obtained using a single LIF resonance. 


\section{Other techniques of ion-beam spectroscopy}

Ion beams find uses in many other applications, three of the main areas involving storage rings (discussed in Sect. 18.3.2), merged beams and studies of the ion-surface interaction at grazing incidence. Merged beam experiments usually study recombination processes involving electrons and atomic or molecular ions (see Sect. 52.1? regarding recombination processes). The advantage of using merged beams is that the time development of the processes may be studied spatially, while maintaining a low relative velocity between the ions and the electrons. This permits measurements at the low energies of importance in studies of Rydberg state formation and in some astrophysical applications. A very different type of experiment studies the ion-surface interaction using a well-collimated ion beam at grazing incidence on a clean, flat surface. Such experiments have revealed very large atomic

orientations [38]. This orientation can be passed on to the nuclei of the atoms via the hyperfine interaction, thus providing a source of oriented nuclei.

\section{SPECTROSCOPY USING ION TRAPS}

A basic purpose of ion traps is to confine ions to the field of view of detectors for time intervals that are longer than the radiative lifetimes of long-lived atomic levels of possible interest. At thermal energies, the ion velocities are large enough to leave a typical detection zone within microseconds. Electrostatic (Kingdon), magnetic (Penning), and radiofrequency (Paul) traps have served for this task for decades (see chapter 73), with recent additions to the armory by electrostatic mirrors of various shapes [39, 40]. Two trap varieties of particular interest for spectroscopy, the electron beam ion trap and the heavy-ion storage ring, will be treated in special sections below.

Collisions with the neutral atoms and molecules of the residual gas cause charge exchange and thus loss of the ion species. Therefore ultrahigh vacuum is of primary importance. Over the last three decades the figure of merit has moved from pressures of about $10^{-8}$ mbar to about $10^{-11}$ mbar. Further improvements can be expected from working with traps at liquid helium temperature; in fact, even ion traps as large as an ion storage ring at Aarhus (see the section below) have been cooled considerably to vary both the vacuum and the amount of blackbody radiation experienced by ions in weakly bound states. 
The energy steps in multiply charged ions are regularly larger than what is available from lasers, at least for excitation from the ground state. Hence single-ion trapping and laser spectroscopic investigation are rarely an option for these ions. Many ions are then needed to provide a sufficiently strong emission signal. The production of quantities of multiply charged ions used to be achieved by electron bombardment of a dilute gas inside the trap volume, or by ablation from a surface. Evidently, this is detrimental to any subsequent measurements, since the residual gas is still present. Precision work like mass spectrometry that exploits the ion cyclotron motion of stored ions, or detailed studies of the radiative processes (including the effects of the interrogating laser field) in ions nowadays employ a sequence of ion traps. In a first trap, the ions of interest are produced and possibly cooled by laser light or other mechanisms, and then, by applying electric fields, the ions of interest are moved to a second trap that works under better vacuum conditions or that can be more finely tuned. In the same sense, a heavy ion storage ring is being fed by an isotopically pure, charge-state selected ion beam. Any loss of ions measured by whatever means thus is proportional to the loss of the ion species of interest.

\section{A. Electron beam ion traps}

Electron beam ion traps (EBIT) make use of the attractive potential of a high-density electron beam as well as of the space charge compensation that is provided by the electron beam to any ion cloud already trapped. Most electron beam ion traps generate the highcurrent density electron beam by feeding the beam from an electron gun into a magnetic field that then compresses and guides the beam. In most cases, superconducting magnets with fields of 3 to $8 \mathrm{~T}$ are being used, and current densities of the order of $10^{4} \mathrm{~A} / \mathrm{mm}^{2}$ are reached.

This high current density corresponds to an electron density of the order of $10^{11} / \mathrm{cm}^{3}$. This low-density environment, roughly comparable to tokamak discharges, is one of the factors that renders the electron beam ion trap a very interesting device for laboratory astrophysics. The magnetic field helps to confine any ion cloud that is produced from the residual gas (or gas bled in) or from injected low charge ions. However, the ions could move away along the field lines, if they were not stopped by potential barriers provided by electrically charged drift tubes. Obviously, the basic design is the same as that of a Penning trap, with the permanent electron beam added. In fact, EBIT with the electron beam on has been called 
to operate in electronic trapping mode [41]; while the same device with the electron beam off ("magnetic trapping mode") still works as a Penning trap. This option of producing an ion cloud with intense electron bombardment and then studying the ions without the electron beam present is the basis for a variety of experiments on charge exchange (CX) reactions and long-lived excited levels [42] (see below).

The first working electron beam ion trap, EBIT-I, has been set up at Livermore [43, 44]. The successful operation instigated an upgrade to SuperEBIT, the first such machine that was able to completely ionize all naturally occurring elements [45]. Mostly based on the Livermore design, some 8 to 10 EBITs are by now either running or under construction around the world. The EBIT operating principles have, for example, been described by Currell [46].

Ionization of ions trapped in the combination of electrical fields proceeds as long as the electron beam energy is high enough to overcome the ionization potential. Thus the highest charge state can be pre-selected by the appropriate choice of the electron beam energy. The technical effort to reach, for example, bare uranium in SuperEBIT is much smaller than when trying the same at an ion accelerator. In both cases the ionization is achieved by frequent energetic collisions of ions with electrons. In SuperEBIT, the ions are (practically) stationary, and the design energy of SuperEBIT, $250 \mathrm{keV}$, is enough to remove even the last electron of uranium. At a heavy-ion accelerator, the electrons are stationary (in a foil target), and the ions are fast. Consequently an ion energy per nucleon that is higher by the proton/electron mass ratio is required - some $500 \mathrm{MeV} / \mathrm{amu}$. Such energetic ion beams are available in only a few large accelerator laboratories, whereas an electron beam ion trap with its auxiliary equipment fits into an office-sized laboratory space. Of course, there are experiments that need specific properties of either fast ion beams or stationary ions, so both types of devices have their specific merit.

The ions in an EBIT are not only stationary in the sense that they are localized in a cloud, and moving either way along the magnetic field with the same probability; their energy (temperature) can also be controlled by the height of the potential barriers. The voltages on the confining drift tubes are usually chosen at a few hundred volts. This makes for barrier potentials + qeU (charge state q, elementary charge e, voltage U) that are higher for highly charged ions than for low-charge state ions. This not only benefits the confinement of highly charged ions directly; light ions (residual gas or purposely bled in gases) may become fully 
ionized by the collisions with the electron beam and by charge exchange, but they still have a larger chance to evaporate from the trap and thus they cool the remaining ion cloud. Under typical conditions the ion cloud may have a temperature of a few $\mathrm{keV}$. This can be lowered by introducing a cooling gas and by lowering the potential barriers. With Cs ${ }^{45+}$ in the trap, this has been demonstrated by reducing the (thermal) Doppler spread of x-ray emission lines until it was smaller than the natural line width of the emitter, thus yielding a measurement of femtosecond level lifetimes from highly resolved x-ray spectra [47].

The other good level lifetime range of an EBIT reaches from a few microseconds (limited by practical switching issues) to many milliseconds. Under direct optical (x-ray, EUV, visible) observation of a spectral feature, the electron beam is used to produce an ion cloud with ions of a desired charge state. When the electron beam is stopped, all direct excitation and prompt emission ceases. Any later photon signal relates to delayed emission from longlived levels, or from excitation by charge transfer collisions (highly charged ions capturing electrons from the residual gas atoms). CX is an important loss mechanism, and the CX signal also serves as a monitor of the number of ions remaining in the trap. Owing to the excellent vacuum in cryogenic EBITs, trapping times of many seconds, if not minutes have been observed [42]. The ion loss rate is the major correction to the apparent decay time of the delayed photon signal. For atomic level lifetimes of a few milliseconds and less, this correction is small (a few percent or less). EBIT lifetime measurements that take this correction into account yield results that agree with those from heavy-ion storage rings [48] and that are, at uncertainties of $0.5 \%$ and less, remarkably consistent with theory in a case that theory can do very well (see fig. 1). This observation can be turned around and interpreted as a demonstration of the reliability of the experimental techniques that then can be applied to more complex cases in which theory evidently has problems (for examples, see $[49,50])$.

An EBIT is an excellent light source for precision spectroscopy of highly charged ions, because it not only gives access to all charge states of all elements, but it does so at low particle densities. Of particular interest to precision spectroscopy have been the ions with a single valence electron ( $\mathrm{Li}, \mathrm{Na}, \mathrm{Cu}$ isoelectronic sequences) that are rather well calculable. Such spectra of ions up to $Z=60$ or 70 have been measured at low-density plasmas like the tokamak, and they have been found to agree well with theory. Higher charge states were then reached in laser-produced plasmas (see chapter 42.2.2?), but the wavelength results seemed 
He sequence M1 transition rate

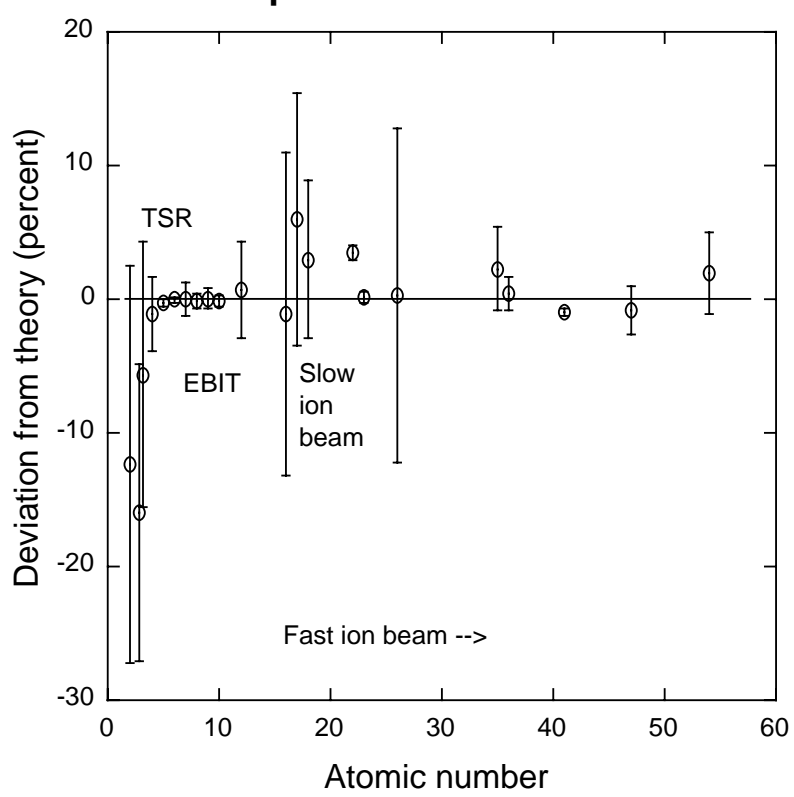

FIG. 1: The lifetime of the $1 \mathrm{~s} 2 \mathrm{~s}{ }^{3} \mathrm{~S}_{1}$ level in the He isoelectronic sequence varies by 15 orders of magnitude from He (about $6000 \mathrm{~s}$ ) to $\mathrm{Xe}^{52+}$ (a few picosedconds). The figure shows the deviation of selected experimental results from calculations, and indicates the dominant experimental techniques for the various lifetime ranges. The consistency of experimental results from heavy-ion storage ring (TSR Heidelberg) and electron beam ion trap (Livermore EBIT-II) with theory in the low-Z range is impressive. Adapted from [49].

to deviate from the theoretical trend that had supported the tokamak results. There also were (very few) high-nuclear charge Z data from fast ion beams. Electron beam ion trap data now have confirmed that the trend of the tokamak data was correct and that theory (including second-order quantum electrodynamics (QED) contributions) describes the $\mathrm{n}=3$ (Na sequence) [51] and $n=4$ levels ( $\mathrm{Cu}$ sequence) [52] well up to $\mathrm{Z}=92$, within the $40 \mathrm{ppm}$ error margin of the EBIT results for the heaviest Cu-like ions. The agreement with theory for the Zn-like ions of the same elements is much poorer. As the QED contributions are rather similar, this is a problem of theory with the computational treatment of two (and more) valence electrons in the same shell.

The atomic systems purportedly under best calculational control are H-like ions, with only one electron at all. In high-Z H-like ions, the lines of highest interest are in the hard $\mathrm{x}$-ray range $(n=1-2)$, or are severely lifetime-broadened $(2 s-2 p)$ and in the EUV. One line is 
in the visible (for a number of ions), where it is accessible to high-resolution spectroscopy, and not even notably broadened, because its upper level has a millisecond-range lifetime: the M1 transition between the hyperfine levels of the ground state. For two isotopes (of Bi and $\mathrm{Pb}$ ), this transition has been induced by laser radiation in a heavy-ion storage ring ([53], see below), and for 5 isotopes (of Re, Ho, and Tl) emission spectroscopy at the SuperEBIT electron beam ion trap [55] was successful. Using the ion cloud (with its cross section largely determined by the electron beam diameter of about $70 \mu \mathrm{m}$ ) as a light source without a further entrance slit, a high-efficiency transmission grating spectrometer, and a position-sensitive detector, lines from two $\mathrm{Tl}$ isotopes were recorded simultaneously [54]. The differential effects of the two nuclei yielded additional information on the charge distribution in the nucleus, on top of the magnetic moment distribution that makes up the dominant effect. These results are required for a better interpretation of parity nonconservation (PNC; see chapter 29) measurements of neutral $\mathrm{Tl}$ atoms, in which the nuclear magnetic moments so far have been treated only as a point dipole. Corresponding measurements are also being done on Li-like ions, and again the most precise data so far (on $\mathrm{Bi}$ ) have been obtained at SuperEBIT.

One of the pertinent problems in astrophysics is the cataloguing of spectral lines in all spectral ranges, both for identifying spectral features and thus learning about the composition of a light source, and for modeling of the light source in order to understand its "operating conditions". Even data bases that claim "practical completeness", however, are found to be grossly incomplete in the EUV and soft-x-ray ranges that have been opened to high-resolution observations by the grating spectrometers on board the Chandra and XMMNewton spacecrafts. Here observations at electron beam ion traps fill in much of the needed data, at comparable quality. Moreover, EBIT as a kind of analog computer does more than provide correct line positions: the spectra show line ratios from a light source with known electron energy (and the option of known temperature by simulating a Maxwellian energy distribution [55]) and particle density. This serves as, both, a check on collisional-radiative models and an immediate data resource for astrophysics.

Most x-ray data on EBITs have been collected using solid state detectors (Si(Li), Ge) that offer high detection efficiency (large solid angle) and signal timing on the microsecond scale, but feature poor spectral resolution. High-resolution instruments like crystal spectrometers are necessary to analyze spectra in any detail; equipped with position-sensitive 
detectors, such instruments do much better than scanning spectrometers in terms of data collection rate and calibration. However, they suffer from the low diffraction efficiency of the crystals. Recently a new device, the microcalorimeter, has started to show its interesting properties. In these devices, small absorbers at mK-temperatures show a measurable temperature increase when absorbing an x-ray photon, and the signal is proportional to the deposited photon energy [56]. The line width of the best devices is below $10 \mathrm{eV}$ (not as good as a crystal spectrometer, but much better than a traditional solid state diode). The sensor pixels can be grouped in arrays to make for a larger area and for cross references among pixels (which helps with calibration and with the rejection of cosmic ray events), and the signal processing is fast enough to permit time resolution on the millisecond range. A spaceflight engineering spare has been used at the Livermore EBITs to study, for example, soft-X-rays of light elements as seen from CX near comets [57], or measure the (10 ms) time constant of the M3 decay in a Ni-like ion $\left(\mathrm{Xe}^{26+}\right)[58]$.

Last, but not least, the well defined and adjustable electron beam energy permits detail studies of the interaction of fast electrons with highly charged ions. This includes, for example, the spectroscopy of dielectronic recombination (DR) resonances, or the exploration of radiative recombination ( $\mathrm{RR}$ ) (see chapter 53). These processes can be investigated up to the highest ion charges, where relativistic and QED contributions (like the Generalized Breit interaction) matter.

\section{B. Heavy-ion storage rings}

With foil-excited ion beams and long level lifetimes, decay curves spread out along the beam, and the signal from a given width of the field of view may drop to the detector background level. The decay lengths of microsecond lifetime levels are on the order of $10 \mathrm{~m}$, and those of millisecond levels of tens of kilometers. In such cases it is advantageous to curve the beam line around on itself, forming a storage ring, in which the ions pass in front of the detector over and over again. Heavy-ion storage rings need excellent vacuum conditions $\left(10^{-11}\right.$ mbar and better $)$ to reach storage times of seconds, minutes, or hours, depending on the electronic structure of the stored ions and on the ion beam energy. The dominant loss processes are electron capture and loss, small angle scattering, and large angle (inelastic) scattering as discussed with any fast ion beams [59], but these contributions are aggravated 
by the much longer path lengths.

Storage rings (for example, TSR Heidelberg, ASTRID Aarhus, CRYRING Stockholm, ESR Darmstadt), with magnetic dipoles and quadrupoles for beam transport and focusing, sort the stored particles by their momentum. The typical ion beam energies range from a few dozen keV total to hundreds of $\mathrm{MeV}$ per nucleon (ESR). Electrostatic storage rings (ELISA Aarhus and more under construction) have only electrical fields and select by particle energy, usually below about $100 \mathrm{keV}$ (for a review, see [60]). They are more suitable for lowcharge heavy ions and ion molecules, including biomolecules, than the magnetic rings that can handle very fast particles. Magnetic storage rings usually have electron cooler sections in which a "cool" electron beam (with a low longitudinal velocity spread, from kinematic compression (see section above) of almost the same velocity as the circulating ion beam is merged with the latter, for a path of a few meters (and is then deflected out again). By scattering among electrons and ions, the momentum spread of the electrons (small) and ions (larger) equilibrates, leaving the ion beam with a narrower momentum distribution and thus cooled. Cooling typically takes a few seconds, it improves the storage behaviour of the ion beam and the energy resolution of, for example, dielectronic recombination (DR) studies (see also chapter 53). For these, the same electron cooler is now tuned to provide electrons at a well defined different velocity. Thus the electron cooler can serve as an electron target, without the complications of a foil target in beam-foil spectroscopy. The difference velocity can be chosen in a wide range, including zero. Extremely low energy collisions are being investigated for the study of DR or for the recombination of molecular radicals. When a beam of molecular ions is injected, storage is long enough to let some of the internal degrees of freedom relax, and then a beam of molecular ions can be extracted that are closer to their ground state.

A cooled ion beam with its narrow velocity distribution is also of interest for laserion interaction studies, offering higher resolution and better signal. Laser-assisted electron capture in the electron cooler, as well as laser spectroscopy on high-lying levels populated by DR, are possible. One of the problems with precision wavelength measurements involving fast ions is always the accurate determination of the velocity, as a step towards determining Doppler corrections. At TSR Heidelberg, a beam of $\mathrm{Li}^{+}$ions was subjected to a laser beam from ahead, tuned to one of the $2 \mathrm{~s}-2 \mathrm{p}$ transitions. A second laser beam from behind probed the position of the Lamb dip in the velocity distribution and thus assured meeting the 
same velocity group of the multi-MeV stored ions. Accurate off-line calibrations of the laser frequencies then permitted a test of the Doppler formula to a relative precison of $2.2 \cdot 10^{-7}$ [61].

The Doppler shift determination in any observation of fast ions requires accurate angle measurements. These are nontrivial, because the detection efficiency of any finite size spectrometer or extended detector may be non-uniform as a function of position or angle. One technique calls for segmented ("granular") x-ray detectors, the strips of which are calibrated individually [62]. Relativity changes the emission pattern seen in the laboratory rest frame to one that favours forward emission. This is beneficial for zero-degree spectroscopy, that is an observation along the ion beam path. At ESR Darmstadt, bare ions captured an electron in the electron cooler section, and resulting x-rays were detected from straight ahead (behind the next dipole magnet that deflected the ion beam). This geometry maximizes the Doppler shift, but minimizes the uncertainty as relating to geometry. Also, after electron capture, the ion in the bending magnet section follows a trajectory that differs from that of the unperturbed ions. The ion can be detected and, in coincidence with the x-ray detector, make for a very clean and charge-specific spectrum. Similar coincidence measurements make it possible to use a low-pressure gas jet target in a high-energy ion storage ring, evaluating only coincidences of x-ray photons and charge-changing events [62]. Fast ions (needed to be so energetic to reach the desired charge state) can also be decelerated in a storage ring, which helps to do systematic checks of the Doppler effect and to work at a lower Doppler shift $[62]$.

As mentioned in section 18.3.1, laser-resonance techniques have been used to find the ground state hyperfine transition in two H-like heavy isotopes. In one of them, also the lifetime was measured, by recording the fluorescence decay from the ion beam after switching off the laser [53]. For lower charge states, one can exploit the excitation that ions carry into the ring from their production in the ion source, or from stripping processes in the injector accelerator [63]. Lifetimes from half a millisecond to several seconds have been measured that way by passive observation [49], with an accuracy of better than $0.2 \%$ in favourable cases. Other techniques use excitation by DR in the ring [64], or laser probing of the remaining metastable level population so that fluorescence is emitted near a photomultiplier detector [65]. With a stored beam of negative ions, even black body radiation may be sufficient to photodetach the weakly bound last electron; the ensuing neutral atom is not deflected at the 
next bending section and leaves the ring to be detected. In summary, lifetime measurements made at heavy-ion storage rings span the enormous range from $10 \mu$ s to about 1 minute.

[1] I. Martinson, Rep. Prog. Phys. 52, 157 (1989).

[2] K. Shima, T. Mikumo, and H. Tawara, At. Data Nucl. Data Tables 34, 357 (1986).

[3] T. Andersen and S. Mannervik, Comments At. Mol. Phys. 16, 185 (1985).

[4] F. G. Serpa and A. E. Livingston, Phys. Rev. A 43, 6447 (1991).

[5] C. F. Bunge, Phys. Rev. Lett. 44, 1450, (1980).

[6] E. J. Knystautas, Phys. Rev. Lett. 69, 2635 (1992).

[7] E. Träbert, P. H. Heckmann, J. Doerfert, and J. Granzow, J. Phys. B: At. Mol. Opt. Phys. 25, L353, (1992)

[8] S. Mannervik, R. T. Short, D. Sonnek, E. Träbert, G. Möller, V. Lodwig, P. H. Heckmann, J. H. Blanke, and K. Brand, Phys. Rev. A 39, 3964, (1989)

[9] E. Träbert, Physica Scripta 48, 699 (1993).

[10] E. Träbert, R. Hutton, I. Martinson Mon. Not. R. Astron. Soc. 227, 27p (1987).

[11] E. Träbert Mon. Not. R. Astron. Soc. 297, 399 (1998)

[12] C. Jupén, P. Bengtsson, L. Engström, and A. E. Livingston, Physica Scripta 64, 329 (2001).

[13] J. O. Stoner and J. A. Leavitt, Opt. Acta 20, 435 (1973).

[14] K.-E. Bergkvist, in Beam Foil Spectroscopy, edited by I. A. Sellin and D. J Pegg (Plenum Press, New York, 1976), p. 719

[15] G. W. F. Drake, Can. J. Phys. 66, 586 (1988).

[16] A. S. Zacharias, A. E. Livingston. Y. N. Lu, R. F. Ward, H. G. Berry, and R. W. Dunford, Nucl. Instrum. Meth. Phys. Res. B 31, 41 (1988).

[17] S. W. Provencher, J. Chem. Phys. 64, 2772, (1976).

[18] D. J. G. Irwin and A. E. Livingston, Comput. Phys. Commun. 7, 95 (1974).

[19] P. H. Heckmann, E. Träbert, H. Winter, F. Hannebauer, H. H. Bukow, and H. von Buttlar, Phys. Lett. A 57, 126 (1976).

[20] E. H. Pinnington, W. Ansbacher, J. A. Kernahan, Z.-Q. Ge, and A. S. Inamdar, Nucl. Instrum. Meth. Phys. Res. B 31, 206 (1988).

[21] R. Geller, App. Phys. Lett. 16, 401 (1970); C. M. Lyneis and T. A. Antaya, Rev. Sci. Instrum. 
61, $221(1990)$.

[22] S. J. Smith, J. A. Lorenzo, S. S. Tayal, and A. Chutjian, Phys. Rev. A 68, 062708 (2003).

[23] J. A. Laming and J. D. Silver, Phys. Lett. A 123, 395 (1987).

[24] G. P. Grandin, D. Hennecart, X. Hussin, D. Lecler, I. Lesteven-Vaisse, and D. Lisfi, Europhys. Lett. 6, 683 (1988).

[25] J. C. Levin, R. T. Short, C.-S. O, H. Cederquist, S. B. Elston, J. P. Gibbons, I. A. Sellin, and H. Schmidt-Böcking, Phys. Rev. A 36, 1649 (1987).

[26] developed largely by the groups of H. Schmidt-Böcking (Frankfurt) and C. L. Cocke (Manhattan, Kansas).

[27] M. Barat, Nucl. Instrum. Meth. Phys. Res. B 9, 364 (1985).

[28] M. H. Prior, R. A. Holt, D. Schneider, K. L. Randall, and R. Hutton, Phys. Rev. A 48, 1964 (1993).

[29] D. Schulze-Hagenest, H. Harde, W. Brand and W. Demtröder, Z. Phys. A 282, 149 (1977); H. Schmoranzer and U. Volz, Physica Scripta T47, 42 (1993).

[30] Y. Baudinet-Robinet, P.-D. Dumont, H.-P. Garnir, and A. El. Himdy, Phys. Rev. A 40, 6321 (1989).

[31] T. J. Scholl, R. Cameron, S. D. Rosner, L. Zhang, R. A. Holt, C. J. Sansonetti, and J. D. Gillaspy, Phys. Rev. Lett. 71, 2188 (1993).

[32] T. P. Dinneen, N. Berrah-Mansour, H. G. Berry, L. Young, and R. C. Pardo, Phys. Rev. Lett. 66, 2859 (1991).

[33] E. J. Myers, J. K. Thompson, E. P. Gavathas, N. R. Clausen, J. D. Silver, and D. J. H. Howie, Phys. Rev. Lett. 75, 3637 (1995).

[34] T. J. Scholl, S. D. Rosner, and R. A. Holt, Can. J. Phys. 76, 39 (1998).

[35] R. C. Rivest, M. R. Izawa, S. D. Rosner, T. J. Scholl, G. Wu, and R. A. Holt, Can. J. Phys.

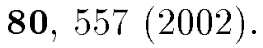

[36] M. L. Gaillard, D. J. Pegg, C. R. Bingham, H. K. Carter, R, L. Mlekodaj, and J. D. Cole, Phys. Rev. A 26, 1975 (1982).

[37] A. Sen, W. J. Childs, and L. S. Goodman, Nucl. Instrum. Meth. Phys. Res. B 31, 324 (1988).

[38] H. J. Andrä, R. Fröhling, H. J. Plöhn, and J. D. Silver, Phys. Rev. Lett. 37, 1212 (1976).

[39] D. Zajfman, O. Heber, L. Vejby-Christensen, I.Ben-Itzhak, M. Rappaport, R. Fishman, and M. Dahan, Phys. Rev. A 55, 1577 (1997). 
[40] H. T. Schmidt, H. Cederquist, J. Jensen, and A. Fardi, Nucl. Instrum. Meth. Phys. Res. B $173,523(2001)$.

[41] P. Beiersdorfer, L. Schweikhard, J. Crespo López-Urrutia, and K. Widmann, Rev. Sci. Instrum. 67, 3818 (1996).

[42] L. Schweikhard, P. Beiersdorfer, E. Träbert, Non-neutral plasma physics IV: Proc. 2001 Int. Workshop on Non-neutral Plasmas, San Diego (CA, USA), edited by F. Anderegg, C. F. Driscoll, and L. Schweikhard, Am. Inst. Phys. Conf. Proc. 606, 174 (2002).

[43] M. A. Levine, R. E. Marrs, J. N. Bardsley, P. Beiersdorfer, C. L. Bennett, M. H. Chen, T. Cowan, D. Dietrich, J. R. Henderson, D. A. Knapp, A. Osterheld, B. M. Penetrante, M. B. Schneider, and J. H. Scofield, Nucl. Instrum. Meth. B 43, 431 (1989).

[44] M. A. Levine, R. E. Marrs, J. R. Henderson, D. A. Knapp, and M. B. Schneider, Physica Scripta T 22, 157 (1988).

[45] R. E. Marrs, S. R. Elliott, and D. A. Knapp, Phys. Rev. Lett. 72, 4082 (1994).

[46] F. J. Currell, in Trapping highly charged ions: Fundamentals and applications, edited by J. Gillaspy, Nova Science Publ., Commack, N.Y., 2001, p. 3

[47] P. Beiersdorfer, A. L. Osterheld, V. Decaux, and K. Widmann, Phys. Rev. Lett. 77, 5353 (1996).

[48] E. Träbert, P. Beiersdorfer, G. Gwinner, E. H. Pinnington, and A. Wolf Phys. Rev. A 66, $052507(2002)$

[49] E. Träbert, Can. J. Phys. 80, 1481 (2002).

[50] P. Beiersdorfer, E. Träbert, and E. H. Pinnington, Astrophys. J. 587, 836 (2003).

[51] P. Beiersdorfer, E. Träbert, H. Chen, M.-H. Chen, M. J. May, and A. L. Osterheld, Phys. Rev. A 67, 052103 (2003).

[52] E. Träbert, P. Beiersdorfer, and H. Chen, Phys. Rev. A 70, 032506 (2004).

[53] I. Klaft, S. Borneis, T. Engel, B. Fricke, R. Grieser, G. Huber, T. Kühl, D. Marx, R. Neumann, S. Schröder, P. Seelig, and L. Völker, Phys. Rev. Lett. 73, 2425 (1994).

[54] P. Beiersdorfer, S. B. Utter, K. L. Wong, J. R. Crespo López-Urrutia, J. A. Britten, H. Chen, C. L. Harris, R. S. Thoe, D. B. Thorn, E. Träbert, M. G. H. Gustavsson, C. Forssén, and A.-M. Mårtensson-Pendrill, Phys. Rev. A 64, 032506 (2001).

[55] D. W. Savin, S. M. Kahn, J. Linkemann, A. A. Saghiri, M. Schmitt, M. Grieser, R. Repnow, D. Schwalm, A. Wolf, T. Bartsch, A. Müller, S. Schippers, M. H. Chen, N. R. Badnell, T. W. 
Gorczyca, and O. Zatsarinny, Astrophys. J. 576, 1098 (2002b).

[56] F. S. Porter, M. D. Audley, P. Beiersdorfer, K. R. Boyce, R. P. Brekosky, G. V. Brown, K. C. Gendreau, J. D. Gygax, S. M. Kahn, R. L. Kelley, C. K. Stahle, A. E. Szymkowiak, Proc. SPIE 4140, 407 (2000).

[57] P. Beiersdorfer, K. R. Boyce, G. V. Brown, H. Chen, S. M. Kahn, R. L. Kelley, M. May, R. L. Olson, F. S. Porter, C. K. Stahle, and W. A. Tillotson, Science 300, 1558 (2003).

[58] E. Träbert, P. Beiersdorfer, et al., (work in progress)

[59] I. S. Dmitriev, V. S. Nikolaev, and Ya. A. Teplova, Phys. Lett. 26A, 122 (1968).

[60] L. H. Andersen, O. Heber, and D. Zajfman, J. Phys. B 37, R57 (2004).

[61] G. Saathoff, S. Karpuk, U. Eisenbarth, G. Huber, S. Krohn, R. Muñoz Horta, S. Reinhardt, D. Schwalm, A. Wolf, and G. Gwinner, Phys. Rev. Lett. 91, 190403 (2003).

[62] Th. Stöhlker, P. H. Mokler, F. Bosch, R. W. Dunford, F. Franzke, O. Klepper, C. Kozhuharov, T. Ludziejewski, F. Nolden, H. Reich, P. Rymuza, Z. Stachura, M. Steck, P. Swiat, and A. Warczak, Phys. Rev. Lett. 85, 3109 (2000).

[63] J. Doerfert, E. Träbert, A. Wolf, D. Schwalm, and O. Uwira Phys. Rev. Lett. 78, 4355 (1997)

[64] Schmidt, H.T., Forck, P., Grieser, M., Habs, D., Kenntner, J., Miersch, G., Repnow, R., Schramm, U., Schüssler, T., Schwalm, D., and Wolf, A., Phys. Rev. Lett. 72, 1616 (1994).

[65] S. Mannervik, Physica Scripta T 105, 67 (2003). 